\title{
comparación resistente de cuatro métodos de refuerzo de pilares de hormigón armado*
}

J. L. RAMIREZ ORTIZ, Prof. Dr. ingeniero industrial J. M. BARCENA DIAZ, ingeniero industrial J. M. FEIJOO GIL, ingeniero industrial

\begin{abstract}
Sinopsis Este trabajo, que es continuación de otro aparecido en INFORMES DE LA CONSTRUCCION, número 272 (julio 1975), estudia el comportamiento resistente de pilares de hormigón armado reforzados en pisos aislados, por otros cuatro procedimientos diferentes:

- Dos de los métodos de refuerzo actualmente utilizados son una mejora de los empleados en el trabajo anterior. Consisten en el recrecido de la sección transversal con hormigón que penetra en cajeras practicadas en el hormigón original o adosado de angulares de acero a las aristas del pilar con capitel de angulares contra el forjado, pero procurando el mejor ajuste entre hormigón y acero mediante resinas.

- Los otros dos métodos son nuevos en nuestra experimentación. Consisten en crear una camisa exterior de hormigón con armadura de zuncho y en la solución metálica en atravesar el pilar con una doble T que transfiera parte de la carga directamente a dos presillas más robustas y, por tan a la transmisión de esfuerzos por el capitel que siempre es algo flexible.
\end{abstract}

\section{INTRODUCCION}

Con este trabajo $y$, continuando otro anterior (INFORMES DE LA CONSTRUCCION, núm. 272, julio 1975), hemos pretendido conocer el comportamiento resistente de cuatro nuevas formas de refuerzo de pilares de hormigón armado.

Al igual que en la ocasión anterior, se ha estudiado dicho comportamiento resistente suponiendo el refuerzo aplicado a pisos aislados, o sea, sin establecer continuidad del refuerzo hacia arriba y hacia abajo en toda la línea del pilar, porque, de esta última forma, la capacidad resistente del refuerzo se aprovecha en su mayor parte, pero a base de un coste bastante más alto.

De los métodos de refuerzo utilizados en esta ocasión, dos son una mejora de los empleados en la anterior experimentación. Consisten en el recrecido de la sección transversal con hormigón que penetra en cajeras practicadas en el hormigón original y en el adosado de angulares de acero a las aristas del pilar con capitel de angulares contra el forjado, pero procurando el mejor ajuste y adherencia entre hormigón y acero por medio de resinas.

\footnotetext{
* Los trabajos se han realizado en los Laboratorios de la Cátedra de Estructuras, de la Escuela Superior de Ingenieros Industriales de Bilbao, y en los Laboratorios de Ensayos e Investigación Industrial "L. J. Torrontegui", anexos, con cargo a una Ayuda a la Investigación concedida por el Ministerio de Educación y Ciencia.
}

Los otros dos métodos presentan diferente planteamiento mecánico. Se basan en crear una camisa exterior de hormigón con armadura de zuncho y en la solución metálica atravesar el pilar con una doble $T$ que transfiera parte de la carga directamente a dos presillas más robustas y, por tanto, dar menos importancia a la transmisión de esfuerzos por el capitel que siempre es algo flexible.

Se llevaron a cabo trabajos de fabricación de los pilares originales, su refuerzo en cuatro formas diferentes y su rotura en prensa, lo cual permitió comparar resultados, dado que era común el hormigón del pilar original con el de los que luego se reforzaban.

De los ensayos se han sacado, además, datos referentes a la distribución de esfuerzos entre pilar original y refuerzo, deformaciones, agrietamientos y forma de rotura.

\section{DIMENSIONES DE PILARES Y REFUERZOS}

\section{Pilar original (fig. 1)}

La sección del pilar original de proyecto ha sido de $25 \times 25 \mathrm{~cm}$ con cuatro redondos de acero liso de $10 \mathrm{~mm}$ de diámetro de armadura longitudinal y estribos de $4,5 \mathrm{~mm}$ de diámetro, separados $15 \mathrm{~cm}$, también en acero liso. Como puede apreciarse, la armadura del pilar es muy reducida, menor incluso que la mínima de normas, 
tratando que fuera la calidad del hormigón la que condicionara principalmente su resistencia a compresión.

Los valores característicos utilizados en el proyecto de este pilar original han sido $180 \mathrm{kp} / \mathrm{cm}^{2}$ en el hormigón y $2.400 \mathrm{kp} / \mathrm{cm}^{2}$ en la armadura. La resistencia realmente obtenida para el hormigón ha sido de $104,8 \mathrm{kp} / \mathrm{cm}^{2}$ (58\% de la de proyecto), por lo que ha quedado justificado el refuerzo.

La carga de proyecto del pilar original $(\mathbb{N})$, de acuerdo con los datos anteriores y las fórmulas de la Instrucción Española EH-73,

$$
\gamma_{\mathrm{n}} \cdot \gamma_{\mathrm{f}} \cdot \mathbf{N}=0,85 \mathbf{b} \cdot \mathbf{h} \cdot 0,9 \cdot \frac{\mathbf{f}_{\mathrm{ck}}}{\gamma_{\mathrm{c}}}-\mathbf{A}_{\mathrm{s}} \cdot \frac{\mathbf{f}_{\mathrm{yk}}}{\gamma_{\mathrm{s}}},
$$

siendo para este caso,

$$
\begin{aligned}
\gamma_{\mathrm{n}} & =1,2, \\
\gamma_{\mathrm{f}} & =1,6, \\
\mathbf{b} & =\mathbf{h}=25 \mathrm{~cm}, \\
\mathbf{f}_{\mathrm{ck}} & =180 \mathrm{kp} / \mathrm{cm}^{2}, \\
\gamma_{\mathrm{c}} & =1,5 \\
\mathbf{A}_{\mathrm{s}} & =4 \times 0,78 \mathrm{~cm}^{2}, \\
\mathbf{f}_{\mathrm{yk}} & =2.400 \mathrm{kp} / \mathrm{cm}^{2}, \\
\gamma_{\mathrm{s}} & =1,15,
\end{aligned}
$$

resulta igual a: $33.275 \mathrm{kp}$.

Para poner de manifiesto el carácter aislado del refuerzo y su acción contra el forjado superior e inferior se ha hecho en la parte superior del pilar un ensanchamiento de $45 \mathrm{~cm}$ de lado y $20 \mathrm{~cm}$ de grueso, que representaba la presencia del forjado. Se ha supuesto, por ser éste el caso más desfavorable, la presencia de un forjado sin vigas salientes, con armadura principal en una sola dirección y compuesta de cuatro redondos, de acero corrugado, de $20 \mathrm{~mm}$ de diámetro: tres en la parte superior y uno en la inferior.

Atendiendo a la condición de simetría del refuerzo respecto al forjado superior e inferior se han ensayado (fig. 1) piezas que tienen $1,60 \mathrm{~m}$ de fuste de pilar, $20 \mathrm{~cm}$ de ensanchamiento representado el forjado y $20 \mathrm{~cm}$ del tamaño primitivo del pilar que simula el pilar del piso superior. Es decir, se ha ensayado prácticamente la mitad del pilar, cogiendo la parte superior, la cual es la que, por posición de las armaduras del forjado, queda más débil desde el punto de vista de rotura de este último por empuje del refuerzo. (Más adelante hacemos algunas observaciones sobre el forjado inferior).

Aunque se han utilizado en el presente trabajo pilares de escuadría pequeña para hacer los ensayos de refuerzos, las conclusiones que se de-

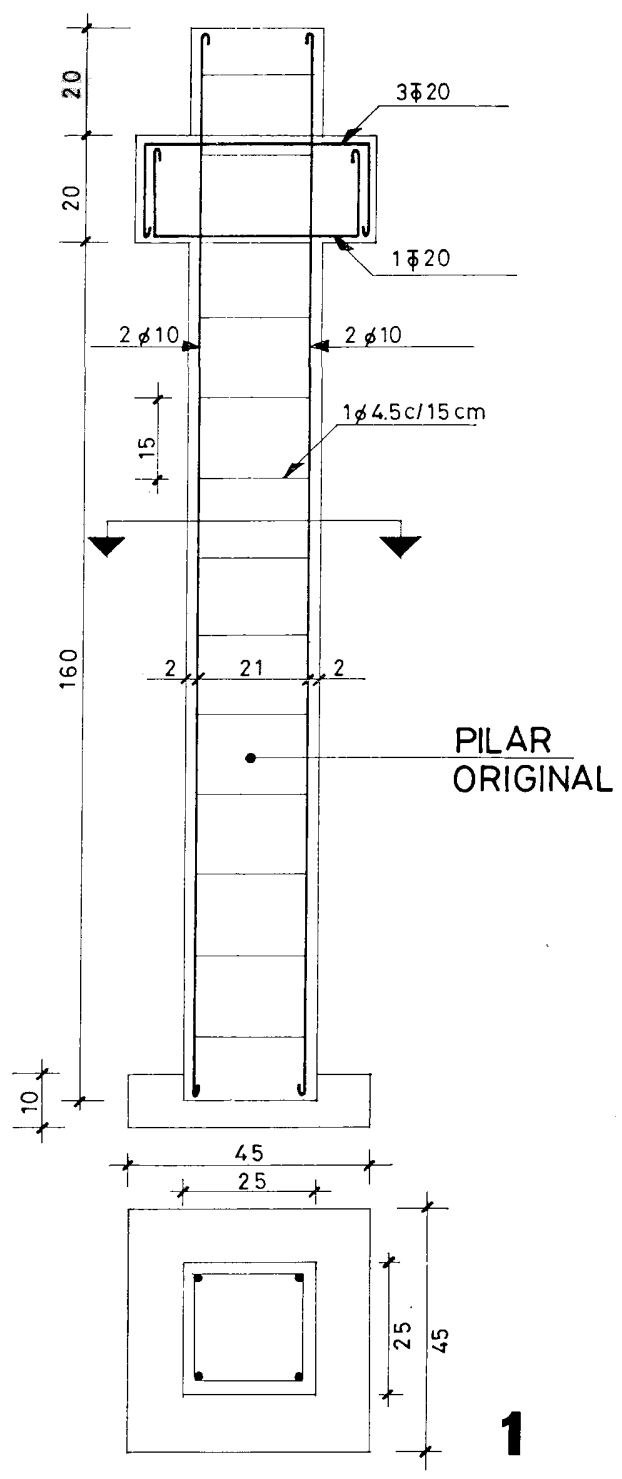

ducen de los ensayos pueden también ser útiles para el estudio de los problemas de refuerzos de pilares de mayor sección, teniendo en cuenta la circunstancia desfavorable de que el espesor de forjado no crece en proporción con la escuadría de los pilares.

\section{Refuerzo en hormigón tipo B (fig. 2) *}

Se ha realizado con el criterio de prescindir por completo del pilar original mal realizado, y que el refuerzo solo, y a compresión simple, fuera capaz de soportar la carga de proyecto del pilar original.

Este tipo de refuerzo en hormigón ha consistido en el regruesamiento de la sección transversal

\footnotetext{
* Los refuerzos tipo A son los analizados en nuestro anterior trabajo publicado en INFORMES DE LA CONSTRUCCION, número 272, julio 1975.
} 


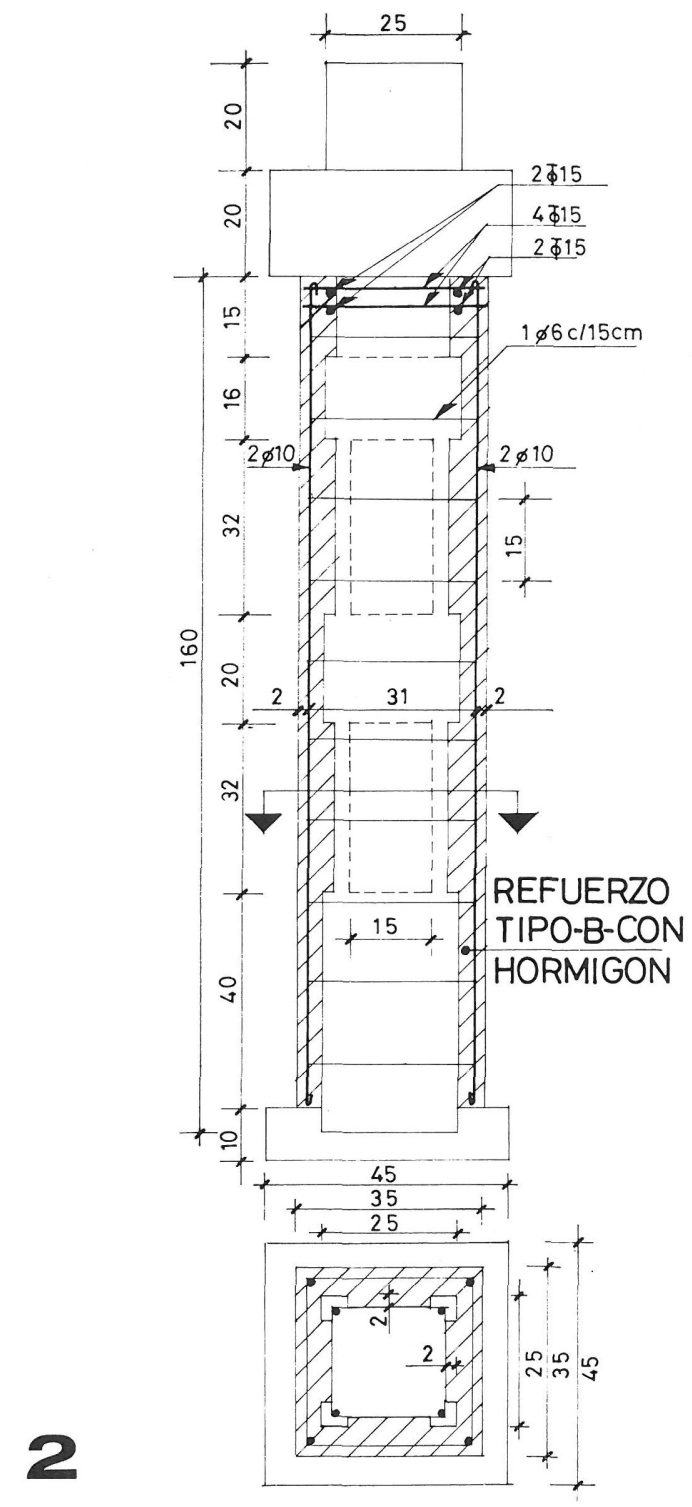

del pilar, pero provocando una íntima unión entre hormigón original y de refuerzo mediante un cajeado convenientemente dispuesto (fig. 3). En realidad es una mejora del utilizado en nuestro trabajo publicado en el número 272 de esta revista. En particular, se ha aumentado la sección transversal del pilar original a $35 \times 35 \mathrm{~cm}$ mediante una capa exterior de un hormigón de calidad nominal $180 \mathrm{kp} / \mathrm{cm}^{2}$ de $5 \mathrm{~cm}$ de grueso, con lo que prácticamente se ha duplicado la sección primitiva de $25 \times 25 \mathrm{~cm}$. Se han colocado en el hormigón añadido cuatro armaduras lisas de $10 \mathrm{~mm}$ de diámetro y estribos lisos de $6 \mathrm{~mm}$ de diámetro cada $15 \mathrm{~cm}$.

Para mejorar la capacidad de transmisión de carga del pilar original al refuerzo se han picado cajeras rectangulares de $2 \mathrm{~cm}$ de profundidad en la superficie del pilar original que suponían un $38 \%$ de su superficie y, además, se picaba igualmente $2 \mathrm{~cm}$ alrededor de todo el pilar en los
$15 \mathrm{~cm}$ inmediatos al forjado, disponiendo de dos barras corrugadas de $15 \mathrm{~mm}$ de diámetro en cada cara (fig. 4). De esta forma la carga del pilar del piso superior pasaba al refuerzo, tanto por el zuncho fuertemente armado creado en la parte contigua del forjado, como por la resistencia a la cortadura que proporcionaba el cajeado previsto.

\section{Refuerzo en hormigón tipo C (fig. 5)}

Ha consistido en zunchar el pilar original picando sus aristas y añadiendo cuatro armaduras lisas de $10 \mathrm{~mm}$ de diámetro en el centro de los lados y colocando una hélice de acero liso de $6 \mathrm{~mm}$ de diámetro unida a las armaduras antiguas y las nuevas con separación de $6 \mathrm{~cm}$ (figuras 6 y 7 ).

La armadura de zuncho se ha calculado para que, junto con las ocho armaduras longitudinales y un núcleo de hormigón de baja calidad (104,8 $\mathrm{kp} / \mathrm{cm}^{2}$ ), permitiera alcanzar al pilar su resistencia de proyecto original de $33.275 \mathrm{kp}$ de carga de servicio. Se ha utilizado para ello la fórmula aditiva de la Instrucción EH-73.

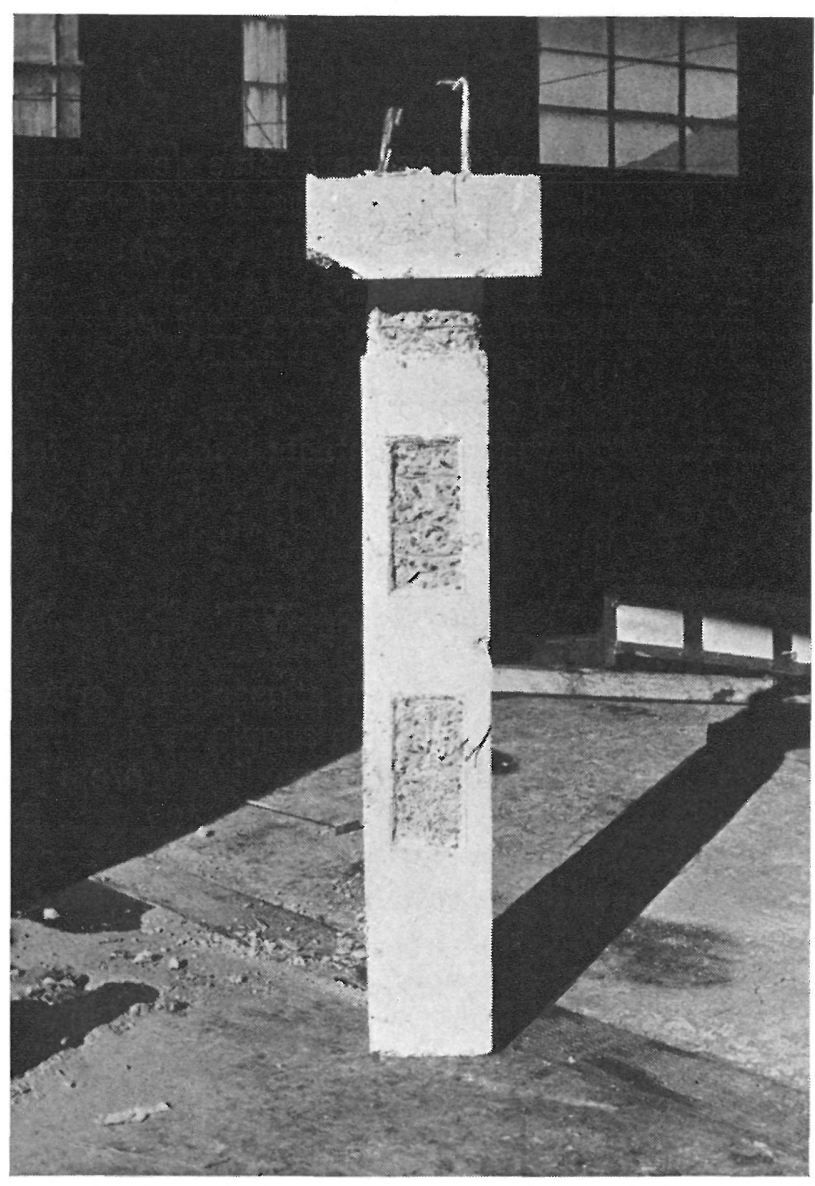

Cajeado en refuerzo en hormigón tipo B. 


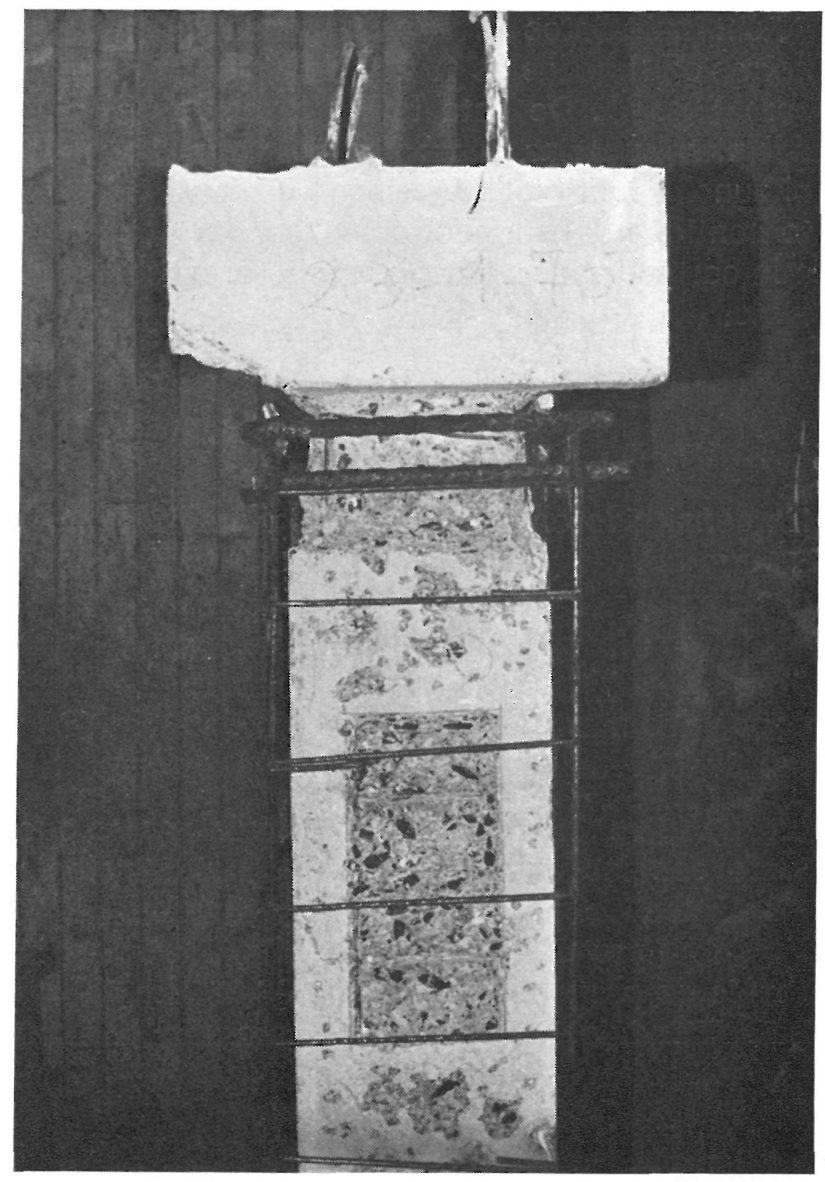

Detalle superior de refuerzo en hormigón tipo $B$.

El pilar, una vez picadas las aristas de la pieza original y colocada la armadura de zuncho, se ha terminado en sección cuadrada de $35 \times 35 \mathrm{~cm}$ con hormigón de $180 \mathrm{kp} / \mathrm{cm}^{2}$ de resistencia nominal. Se ha dejado una holgura de $1 \mathrm{~cm}$ entre esta zona de zuncho añadida y la parte inferior del forjado para evitar transmisión directa longitudinal de carga por contacto con dicho forjado.

\section{Refuerzo metálico tipo B (fig. 8)}

El refuerzo ha consistido en adosar a las cuatro aristas del pilar de hormigón angulares de acero de $55 \times 55 \times 6 \mathrm{~mm}$ unidos mediante presillas soldadas de $80 \times 6 \mathrm{~mm}$ separadas $400 \mathrm{~mm}$. Es también una mejora del descrito en el número 272 de esta revista, ya que los cuatro trozos de angulares que forman el capitel bajo el forjado llevan unos pitones soldados por su parte interior que entran en una zona de $50 \mathrm{~mm}$ de altura que penetra hasta la armadura, que se ha rellenado con un mortero especial de resina, produciendo un total ajuste entre los angulares del capitel y el fuste del pilar y parte baja del forjado.
Este relleno se ha hecho por dos agujeros que atravesaban el espesor del forjado y desembocaban en dicha cavidad. Con todo ello se transmite más directamente la carga del fuste del pilar a la estructura metálica y se pone menos en juego la cortadura del forjado.

La sección transversal de los angulares se ha calculado por las fórmulas correspondientes de cálculo de estructura metálica de la Norma MV103, de manera que fuera capaz por sí sola de resistir la carga de proyecto del pilar original.

\section{Refuerzo metálico tipo C (fig. 9)}

Como antes, el refuerzo ha consistido en adosar a las cuatro aristas angulares de acero de $55 \times 55 \times 6 \mathrm{~mm}$ unidas mediante presillas de $80 \times 6 \mathrm{~mm}$ separadas $40 \mathrm{~cm}$.

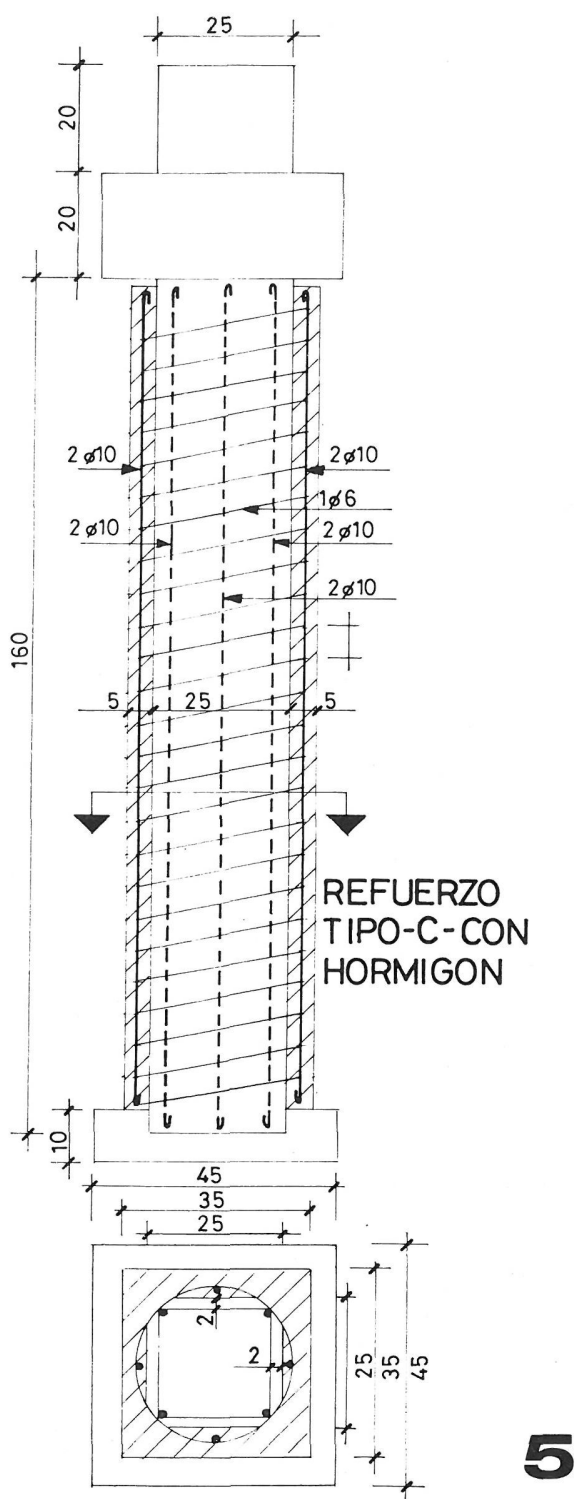




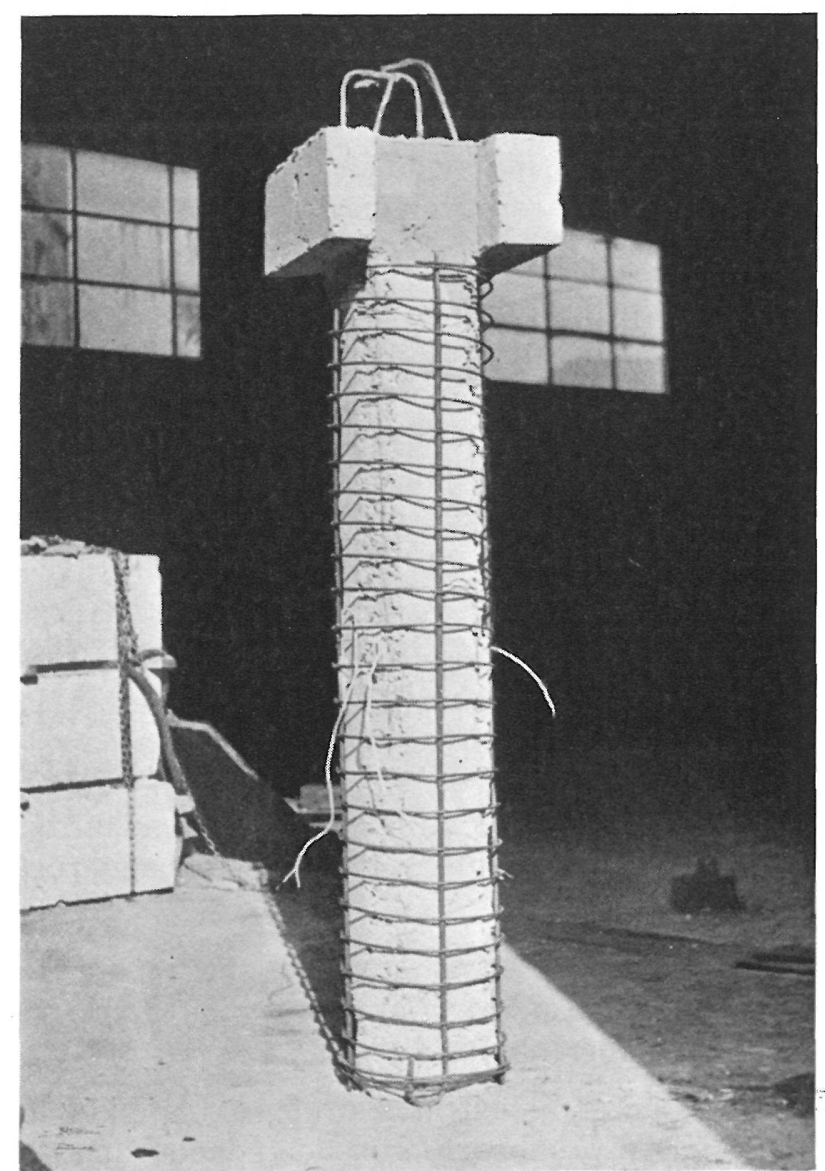

Refuerzo en hormigón tipo C.

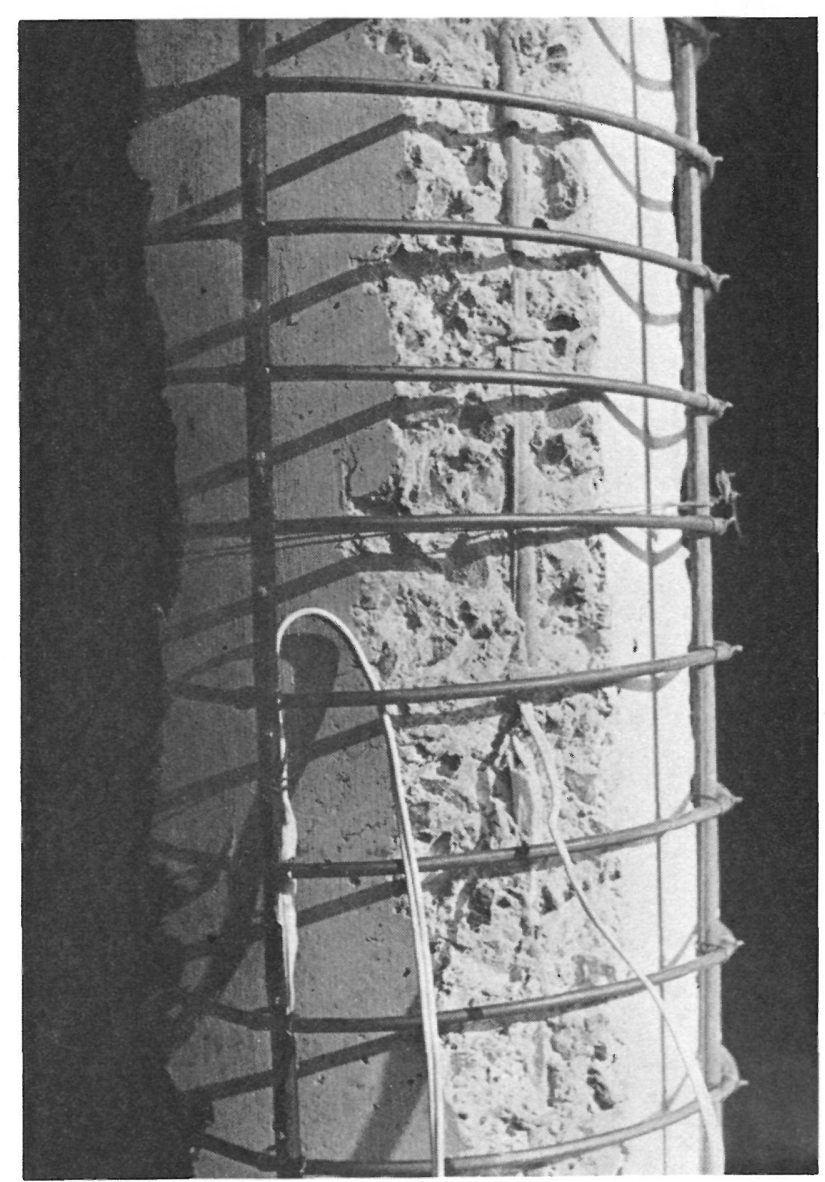

Refuerzo en hormigón tipo C (detalle).

Sin embargo, en esta ocasión se ha querido mejorar la transmisión directa de la carga del pilar del piso superior a los angulares de refuerzo, y para ello se ha cruzado, atravesando el pilar original, un trozo de doble $\mathrm{T}$ que se ha apoyado en dos presillas especiales soldadas a los angulares de refuerzo (fig. 10). Posteriormente, se ha rellenado el agujero producido en el pilar original para colocar la doble $\mathrm{T}$ mediante mortero de resina especial, con lo que ha quedado todo ligado y rellenos los huecos, en particular entre la parte inferior del forjado y superior de la doble $\mathrm{T}$.

El dimensionamiento de esta parte superior del refuerzo se ha hecho absorbiendo por contacto, pilar superior, forjado, doble $\mathrm{T}$, el $42 \%$ de la carga. Se ha tenido en cuenta la mejora en el comportamiento resistente del hormigón por transmisión en área reducida (1/3) de su sección transversal. Se ha calculado para esta carga la flexión del trozo de doble $T$ entre presillas,
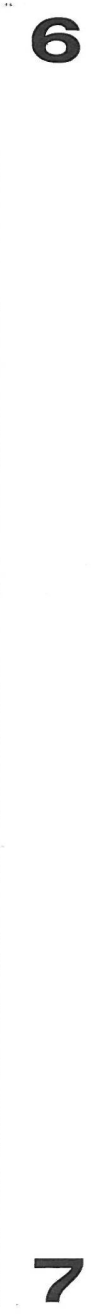

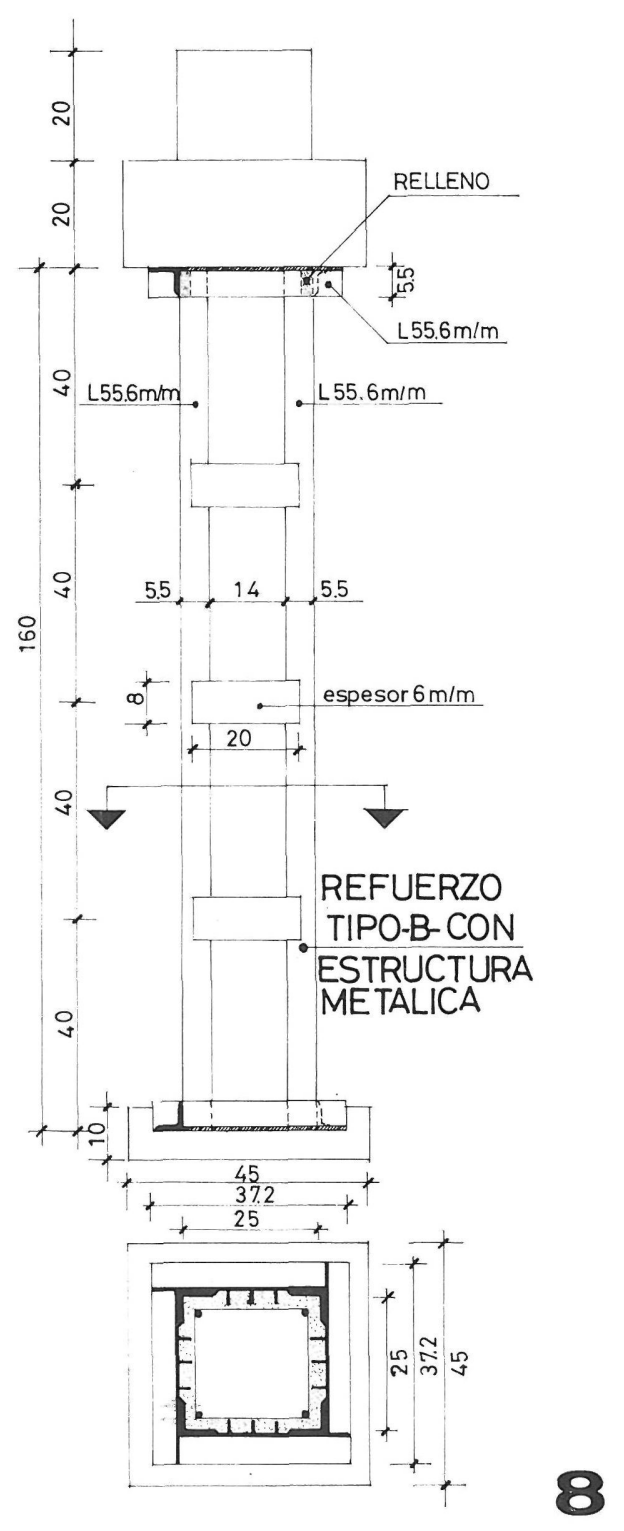




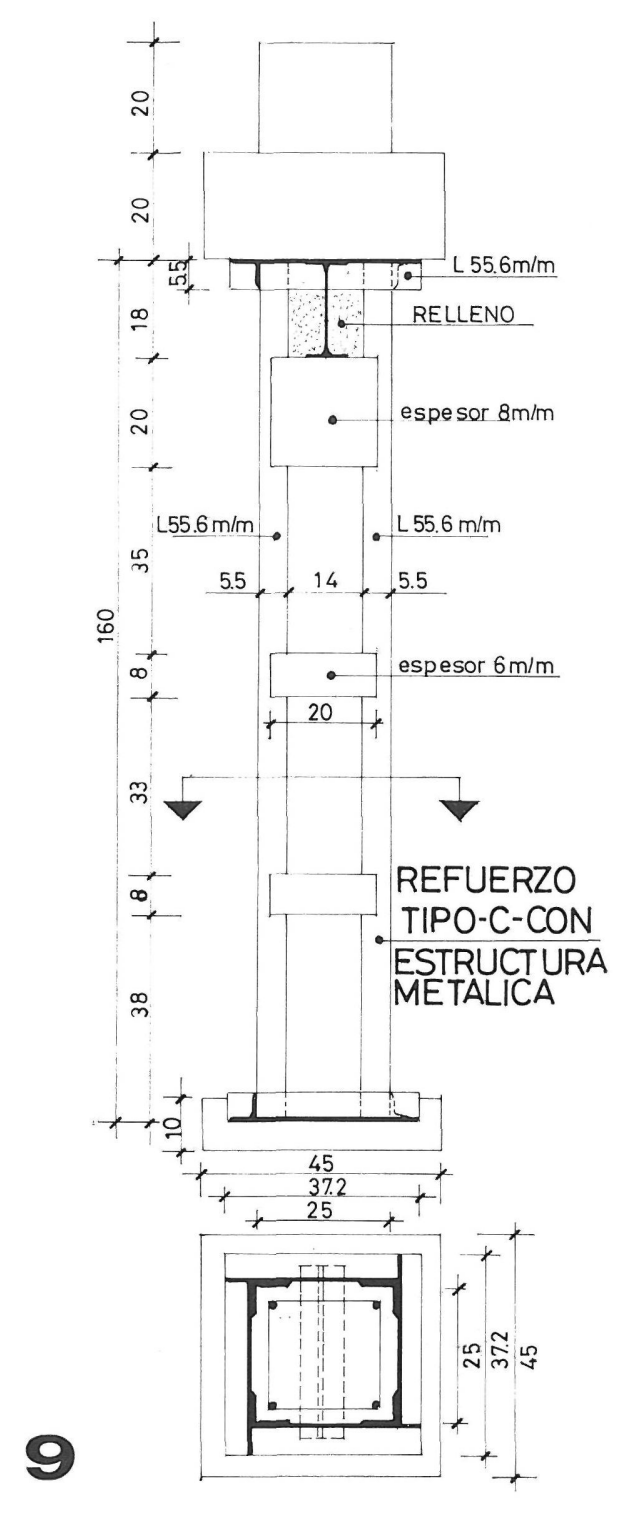

la medida de éstas y su unión con los angulares longitudinales. Con ello han resultado IPN de $18 \mathrm{~cm}$ y presillas de apoyo de $200 \times 8 \mathrm{~mm}$.

Además se ha colocado el clásico capitel de angulares soldados entre sí, a los angulares longitudinales y a la doble $\mathrm{T}$.

\section{MATERIALES DE LOS PILARES DE HORMIGON Y DE LOS REFUERZOS}

El conjunto de la experimentación realizada ha consistido en:

a) Preparación de 6 series de tres pilares idénticos cada una.

b) En el refuerzo de dos pilares de cada serie, dejando el tercero para comparación. Con los 12 pilares a reforzar se han utilizado cuatro métodos diferentes de refuerzo; es decir, que se han empleado tres pilares por método.

c) En el ensayo de todos los pilares a compresión simple, midiendo cargas, acortamientos $\mathrm{y}$ tensiones en armaduras y angulares por medio de extensómetros óhmicos.

El hormigón de los pilares originales, junto con el ensanchamiento del forjado se confeccionó con árido calizo de machaqueo de $30 \mathrm{~mm}$ de tamaño máximo y dosificación de $200 \mathrm{~kg} / \mathrm{m}^{3}$ de cemento P-350 y relación agua/cemento de 1,23, con objeto de obtener un "mal" hormigón de baja resistencia que en nuestro caso resultó de $104,8 \mathrm{kp} / \mathrm{cm}^{2}$.

La presencia del pilar del piso superior se materializó posteriormente hormigonando sobre la parte superior del forjado una cabeza de hormigón de $25 \times 25 \mathrm{~cm}$ de sección y $20 \mathrm{~cm}$ de altura, para el que se utilizó una dosificación de $375 \mathrm{~kg} / \mathrm{m}^{3}$ de cemento y una relación agua/cemento de 0,7 .

Finalmente, se ejecutó una pequeña zapata de hormigón en la parte inferior del pilar para mejorar el asiento sobre la prensa.

Las armaduras y estribos utilizados a todo lo largo de las pruebas fueron de redondo liso de

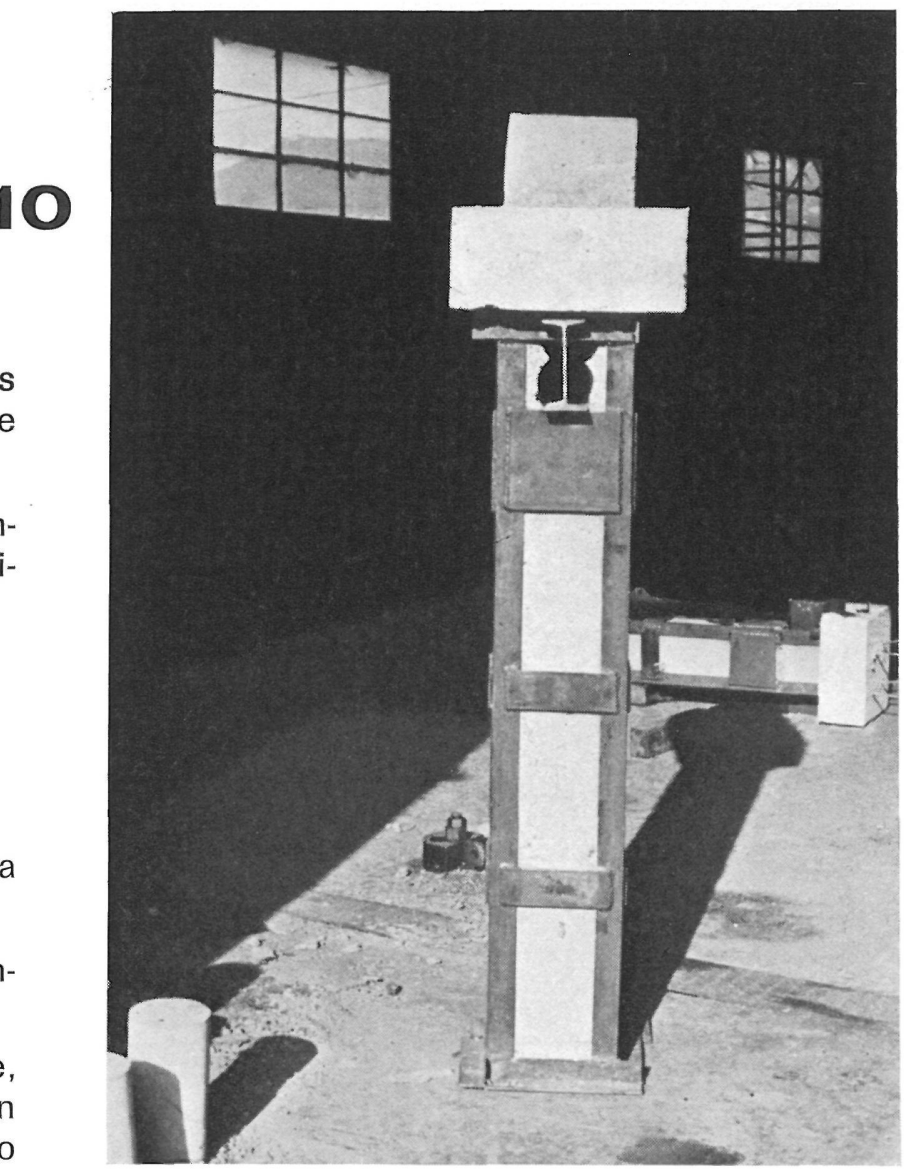

Refuerzo con estructura metálica tipo C. 
acero dulce corriente AE $24 \mathrm{~L}$ de $\mathbf{f}_{\mathrm{yk}}=2.400$ kilopondios/centímetro cuadrado, con excepción del corrugado utilizado en la zona del forjado, que fue estirado en frío, del tipo $A E 42 F$ de $\mathbf{f}_{\mathrm{yk}}=$ $=2.400 \mathrm{kp} / \mathrm{cm}^{2}$.

Damos ahora información del material empleado en la ejecución de los refuerzos.

El hormigón utilizado en los tipos de refuerzo con este material debía de cumplir dos condiciones: en primer lugar colar bien en el reducido espacio de $5 \mathrm{~cm}$ de espesor que tenía la pared de los refuerzos; además, tener una resistencia de $180 \mathrm{kp} / \mathrm{cm}^{2}$ que es la original de proyecto.

Todo ello se ha conseguido con una mezcla de $315 \mathrm{~kg} / \mathrm{m}^{3}$ de cemento P-350 y árido calizo, pero de tamaño máximo de $12 \mathrm{~mm}$ y relación agua/ cemento de 0,88 .

Con objeto de aproximarnos a las mediciones de la obra, este hormigón se vertió por unos orificios practicados en la zona que simula el forjado, con los pilares en posición vertical, vibrándose convenientemente y no apreciándose después coqueras o defectos de compactación. Se apiconó previamente el pilar primitivo para aumentar la adherencia entre el hormigón antiguo y el nuevo.

Todo el material utilizado en el refuerzo en estructura metálica, angulares, chapa y doble $T$ fue de la calidad A 37b de la Norma MV 102, con un límite elástico mínimo de $2.400 \mathrm{kp} / \mathrm{cm}^{2}$.

El control de las calidades de los hormigones, tanto del pilar original como de los dos tipos de refuerzo y de las cabezas y pies, se realizó mediante probetas cilíndricas de $15 \times 30 \mathrm{~cm}$, que en la fecha de ensayo de los mismos pilares dieron los resultados recogidos en el cuadro 1.

\section{ENSAYOS DE ROTURA DE PILARES}

Una vez realizados los refuerzos se dispuso para el ensayo de 6 series de pilares, formada cada una por un pilar original sin reforzar y otras dos unidades reforzadas por dos de los cuatro procedimientos de refuerzo antes descritos.

Los tres pilares de cada serie se ensayaron en la misma fecha utilizando una prensa de $250 \mathrm{t}$, capaz para pilares de $2 \mathrm{~m}$ de alto y $45 \times 45 \mathrm{~cm}$ de sección y provista de rótula.

La edad del hormigón en el momento de la rotura fue de 100 días para el pilar primitivo y de 50 días para el del refuerzo, con objeto de aproximarnos a las condiciones de obra.
Los resultados están recogidos en el cuadro 2, en el que además se incluyen las relaciones entre la resistencia de los pilares reforzados por los cuatro procedimientos con la resistencia del pilar sin reforzar, permitiendo apreciar la mejora resistente que proporcionan.

También son de mucho interés las relaciones entre las resistencias de los pilares reforzados y la teórica que hubiera tenido un pilar hecho con hormigón de la resistencia correcta. Esta carga de rotura teórica se puede calcular por la fórmula de resistencia a compresión de la EH-73, sin emplear el coeficiente de fatiga debido a que los ensayos de rotura que hemos hecho pueden considerarse como ensayos rápidos:

$$
\mathbf{N}_{\mathrm{r}}=\mathbf{b} \cdot \mathbf{h} \cdot 0,9 \mathbf{f}_{\mathrm{ck}}+\mathbf{A}_{\mathrm{s}} \cdot \mathbf{f}_{\mathrm{yk}},
$$

siendo:

$$
\begin{aligned}
\mathbf{b}=\mathbf{h} & =25 \mathrm{~cm}, \\
\mathbf{f}_{\mathrm{ck}} & =180 \mathrm{kp} / \mathrm{cm}^{2}, \\
\mathbf{A}_{\mathrm{s}} & =4 \times 0,78 \mathrm{~cm}^{2} \\
\mathbf{f}_{\mathrm{yk}} & =2.400 \mathrm{kp} / \mathrm{cm}^{2} .
\end{aligned}
$$

Resulta: $\mathbf{N}_{\mathrm{r}}=108.740 \mathrm{kp}$.

Los valores de las relaciones para todos los pilares ensayados figuran en el cuadro 3.

De los cuadros 2 y 3 se deduce que, si bien por todos los procedimientos se alcanza, desde el punto de vista de valores medios, prácticamente el valor teórico de rotura en ensayo rápido, es el procedimiento de refuerzo metálico tipo $\mathrm{C}$, con doble $\mathrm{T}$ insertada bajo el forjado, el que destaca por su clara mayor eficacia.

\section{FORMA DE LAS ROTURAS}

\section{a) Pilares sin reforzar}

En estos pilares las roturas se produjeron en general en la parte superior del fuste de los pilares, a 40 ó $50 \mathrm{~cm}$ de la parte inferior del forjado. Es lógico debido a la segregación del hormigón y la menor compactación de esta zona respecto a la inferior (fig. 11).

\section{b) Pilares reforzados con hormigón}

En los tipos de refuerzo B y C la rotura se ha producido fundamentalmente por el forjado original de baja calidad bajo la presión que le transmitía el dado de hormigón que representa al pilar superior. Próximo a la rotura aparecían algunas grietas verticales que afectaban a las aristas del fuste reforzado en la proximidad de la parte inferior del forjado (fig. 12). 
CUADRO 1

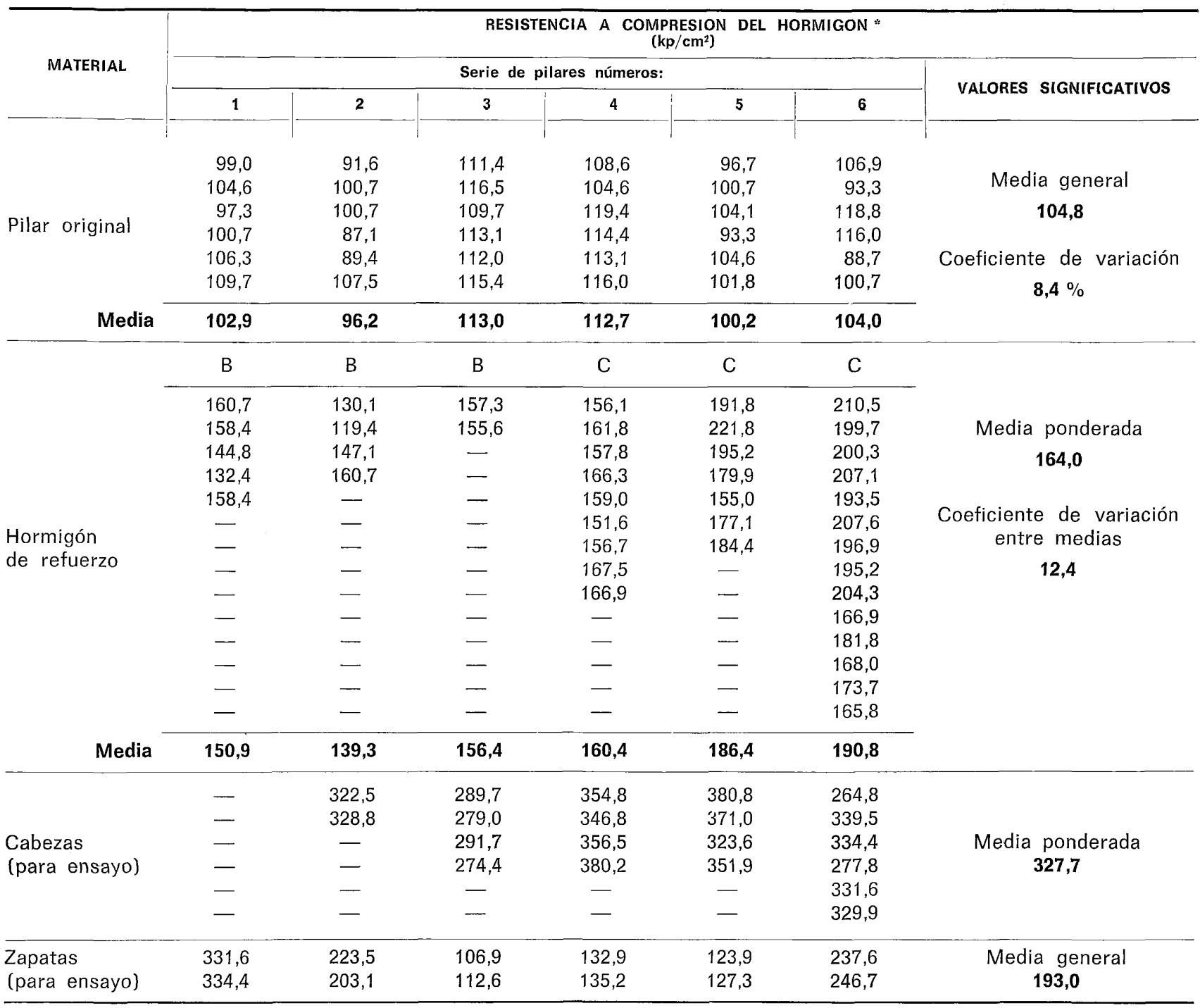

" Probetas correspondientes al pilar original conservadas en agua.

Probetas correspondientes al hormigón de refuerzo conservadas al aire.

Probetas correspondientes a cabezas y zapatas conservadas al aire.

CUADRO 2

\begin{tabular}{|c|c|c|c|c|c|c|c|c|}
\hline \multirow{3}{*}{\multicolumn{2}{|c|}{ ESTADO DEL PILAR }} & \multicolumn{7}{|c|}{ RESISTENCIA DE LOS PILARES SIN Y CON REFUERZO (kp) } \\
\hline & & \multicolumn{6}{|c|}{ Serie de pilares números: } & \multirow{2}{*}{ Media } \\
\hline & & 1 & 2 & 3 & 4 & 5 & 6 & \\
\hline Sin reforzar (I) & & 76.370 & 65.740 & 63.460 & 60.420 & 66.750 & 73.080 & 67.636 \\
\hline \multirow{2}{*}{ Refuerzo en hormigón } & $\mathrm{B}(\mathrm{II})$ & 113.060 & 103.970 & 111.560 & 一 & - & - & 109.530 \\
\hline & $C(I I I)$ & - & - & - & 100.430 & 103.470 & 113.060 & 105.653 \\
\hline \multirow{2}{*}{ Refuerzo metálico } & $\mathrm{B}$ (IV) & - & - & - & 113.590 & 103.470 & 116.120 & 111.060 \\
\hline & $C(V)$ & 134.830 & 126.240 & 127.740 & 一 & - & 一 & 129.603 \\
\hline \multicolumn{2}{|l|}{ Relación (II) / (I) } & 1,48 & 1,58 & 1,75 & - & - & - & 1,60 \\
\hline \multicolumn{2}{|l|}{ Relación (III)/(I) } & - & - & - & 1,66 & 1,55 & 1,54 & 1,58 \\
\hline \multicolumn{2}{|l|}{ Relación (IV) / (I) } & - & - & - & 1,88 & 1,55 & 1,58 & 1,67 \\
\hline \multicolumn{2}{|l|}{ Relación (V)/(I) } & 1,76 & 1,92 & 2,01 & - & - & - & 1,89 \\
\hline
\end{tabular}


CUADR 3

\begin{tabular}{|c|c|c|c|c|c|c|c|}
\hline \multirow{3}{*}{ TIPO DE RELACION } & \multicolumn{7}{|c|}{ RELACIONES DE RESISTENCIA DE PILARES } \\
\hline & \multicolumn{6}{|c|}{ Serie de pilares números: } & \multirow{2}{*}{ Media } \\
\hline & 1 & 2 & 3 & 4 & 5 & 6 & \\
\hline Sin reforzar & \multirow{2}{*}{0,70} & \multirow{2}{*}{0,60} & \multirow{2}{*}{0,58} & \multirow{2}{*}{0,55} & \multirow{2}{*}{0,61} & \multirow{2}{*}{0,67} & \multirow{2}{*}{0,62} \\
\hline 108.740 & & & & & & & \\
\hline Refuerzo en hormigón (B) & 1,04 & 0,95 & 1,02 & - & - & \multirow{2}{*}{-} & \multirow[t]{2}{*}{1,00} \\
\hline 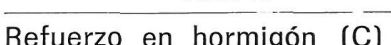 & \multirow[b]{2}{*}{-} & \multirow[b]{2}{*}{-} & \multirow[b]{2}{*}{-} & \multirow[b]{2}{*}{0,92} & & & \\
\hline$\frac{\text { Reruerzo en normigon (C) }}{108.740}$ & & & & & 0,95 & 1,04 & 0,97 \\
\hline Refuerzo metálico (B) & \multirow{2}{*}{-} & \multirow{2}{*}{ - } & \multirow{2}{*}{-} & \multirow{2}{*}{1,04} & \multirow{2}{*}{0,95} & \multirow{2}{*}{1,07} & \multirow{2}{*}{1,02} \\
\hline 108.740 & & & & & & & \\
\hline Refuerzo metálico (C) & \multirow{2}{*}{1,24} & \multirow{2}{*}{1,16} & \multirow{2}{*}{1,17} & \multirow{2}{*}{-} & \multirow{2}{*}{ - } & \multirow{2}{*}{ - } & 110 \\
\hline 108.740 & & & & & & & 1,19 \\
\hline
\end{tabular}

\section{c) Pilares reforzados con estructura metálica}

En el refuerzo tipo $B$ las primeras fisuras aparecen en la parte superior del fuste del pilar de hormigón, como en el caso de un pilar sin refuerzo, absorbiéndose todavía a partir de ese momento algo más carga y terminando rompiendo por expansión del forjado (fig. 13).

En el refuerzo tipo $\mathrm{C}$, debido a la mejor transferencia de carga de la cabeza de hormigón al

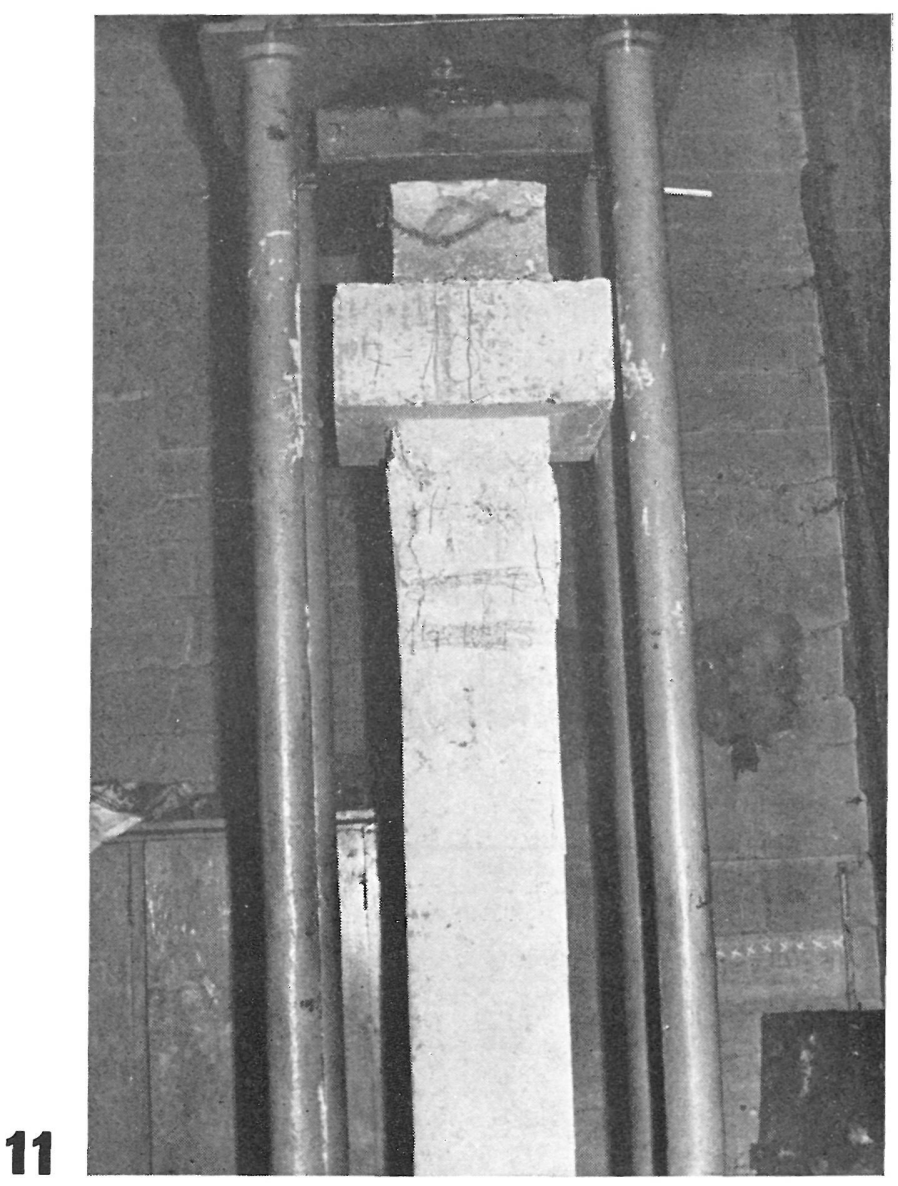

refuerzo metálico mediante la doble T insertada, la rotura se localiza únicamente en el forjado de baja calidad. La mejora respecto a los otros procedimientos puede estar en el efecto de zuncho para la parte inferior del forjado que supera la presencia del ala superior de la doble T, además de los cuatro angulares horizontales (fig. 14).

\section{DEFORMABILIDAD DEL PILAR ORIGINAL Y DE LOS REFORZADOS}

Utilizando valores medios se han representado en la figura 15 las curvas carga-deformación de los cinco tipos de pilares ensayados. Aun cuando no se ha utilizado una instrumentación de medida de deformación muy precisa, estimamos que las curvas presentadas son suficientemente fiables por su coincidencia con valores deducidos de los extensómetros óhmicos colocados en armaduras y angulares.

Como observaciones más importantes a partir de las curvas pueden hacerse las siguientes:

- Se advierte la menor deformabilidad de los pilares reforzados respecto al de baja calidad sin reforzar y en particular la menor deformabilidad de los reforzados con hormigón.

- Para la carga de servicio (33.275 kp), la deformabilidad de los refuerzos en hormigón es el $50 \%$ de la correspondiente al refuerzo metálico. No se nota para este valor de la carga que haya diferencia de deformabilidad entre las dos soluciones de refuerzo, ya sea en hormigón ya sea en estructura metálica.

- Para los valores de la rotura las soluciones de hormigón zunchado y de insertado de doble $T$ son, dentro de cada material de refuerzo, las que presentan menor deformación. 


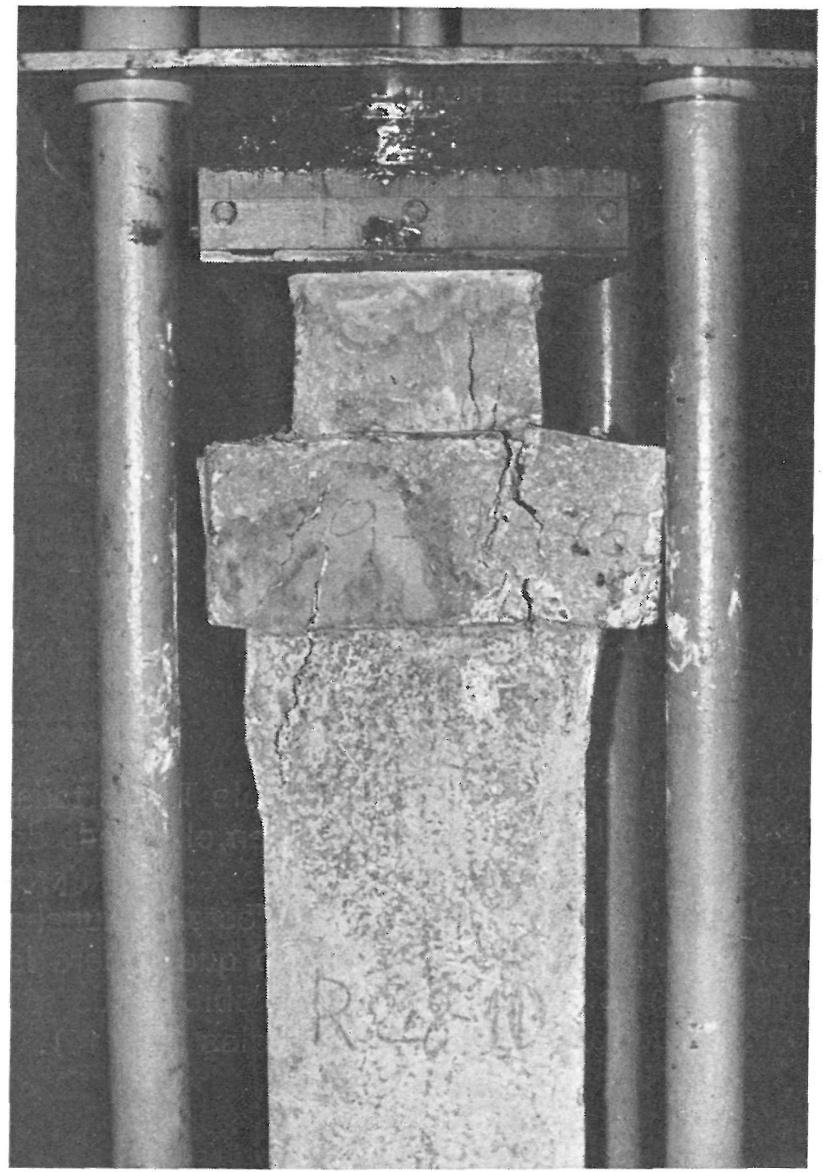

Rotura de pílar reforzado en hormigón tipo B

\section{REPARTO DE CARGAS ENTRE PILAR ORIGINAL Y REFUERZO}

En el cuadro 4 se reflejan los porcentajes de la carga total soportados por la parte original del pilar y el refuerzo para diferentes estados de carga.

Han sido deducidos a partir de los extensómetros óhmicos pegados a las armaduras de los pilares originales, a las de los refuerzos de hormigón y a los angulares de acero laminado de las soluciones metálicas.

Se observa en el cuadro 4 que en los pilares reforzados con una solución de hormigón el refuerzo va perdiendo participación al aumentar

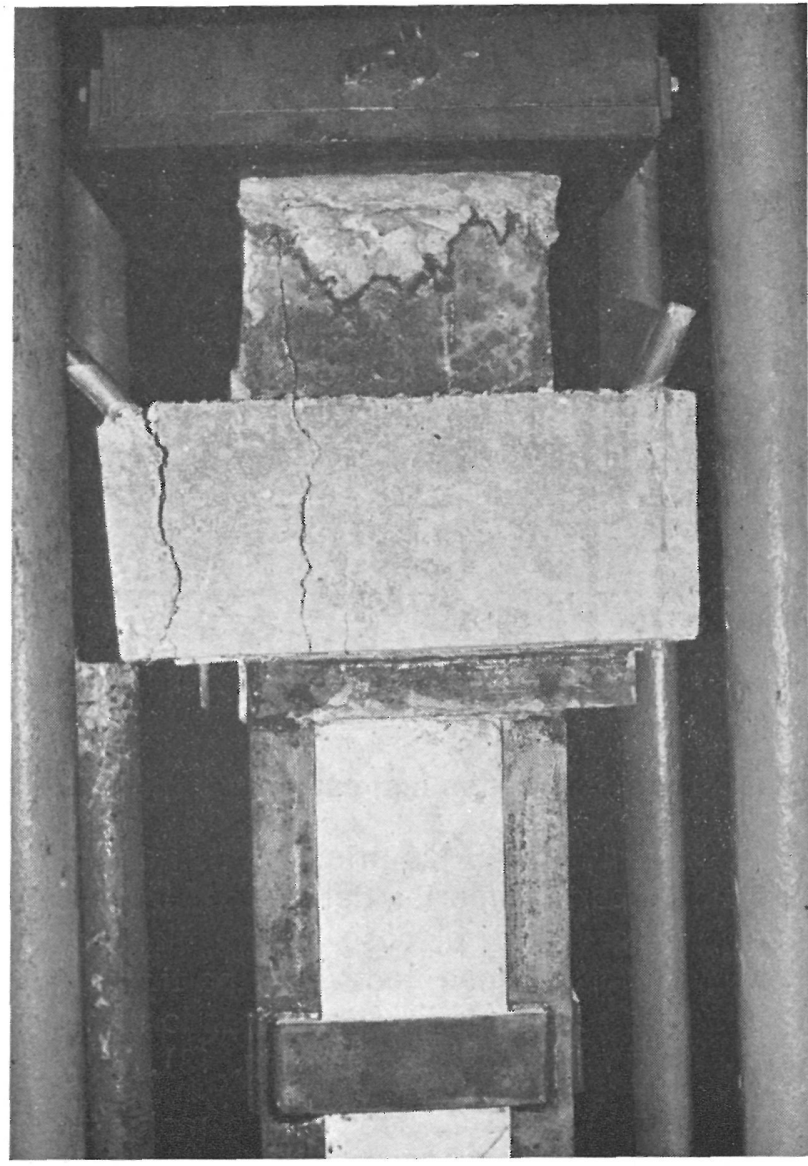

Rotura de pilar reforzado con estructura metálica tipo B.

la carga, ocurriendo al revés en los refuerzos con solución metálica.

Claro está que lo expuesto ha sido basándose en ensayos rápidos que no permiten poner en juego la diferencia en el comportamiento plástico diferido relativo del hormigón de mala calidad primitivo y cualquier tipo de refuerzo.

Estimamos que al ceder plásticamente el hormigón interior al refuerzo se pondrá más en juego la cortadura del forjado que tiene un valor pequeño y que las relaciones resistencia pilares reforzados/resistencia pilar original, bajo cargas permanentes, serán menores de las obtenidas en los ensayos rápidos reflejados en el cuadro 3 .

C U A D R O 4

\begin{tabular}{|c|c|c|c|c|c|c|}
\hline \multirow{3}{*}{ TIPO DE REFUERZO } & \multicolumn{6}{|c|}{$\%$ DE LA CARGA TOTAL SOPORTADA EN LA FASE DE: } \\
\hline & \multicolumn{2}{|c|}{ CARGA DE SERVICIO } & \multirow{2}{*}{\multicolumn{2}{|c|}{ DOBLE CARGA SERVICIO }} & \multicolumn{2}{|c|}{ ROTURA PIEZA } \\
\hline & Original & Refuerzo & & & Original & Refuerzo \\
\hline Hormigón B & 48 & 52 & 53 & 47 & 61 & 39 \\
\hline Hormigón C & 43 & 57 & 46 & 54 & 55 & 45 \\
\hline Metálica B & 62 & 38 & 56 & 44 & 55 & 45 \\
\hline Metálica C & 66 & 34 & 59 & 41 & 52 & 48 \\
\hline
\end{tabular}




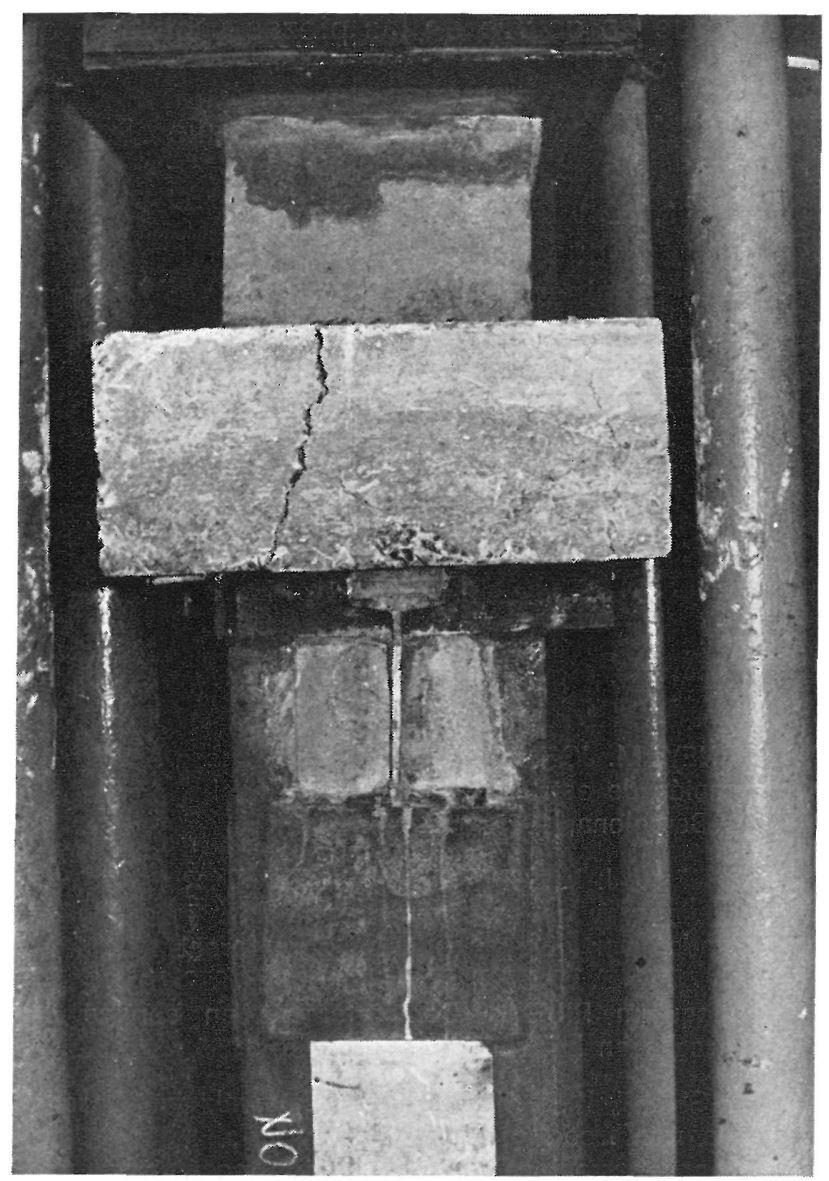

Rotura de pilar reforzado con estructura metálica tipo C.

\section{CONCLUSIONES}

En este trabajo hemos pretendido comparar cuatro formas de refuerzo de pilares, con el pensamiento puesto en que los refuerzos pudieran realizarse en pisos aislados.

De acuerdo con los cuadros 2 y 3 hemos visto que en las cuatro modalidades de refuerzo se ha alcanzado, desde el punto de vista de resultados medios, el valor de la resistencia proyectada para el pilar original. Probablemente, desde el punto de vista de valores característicos o con un cierto grado de confianza, es el de la doble $\mathrm{T}$ atravesada el único sistema que lograría valor superior a la unidad para la relación resistencia reforzado/carga de proyecto.

Hay que tener en cuenta, sin embargo, que la relación bajará, creemos, cuando se trate de cargas permanentes.

Este método de refuerzo nos parece muy interesante, pues no se apoya en el corte del forjado o de las vigas, sino que coge de forma centrada la carga del pilar superior y la transmite al pilar inferior.

En un caso práctico, teniendo en cuenta el aumento de resistencia que presenta el hormigón por compresión localizada, habría que dimensionar el calibre de la doble $\mathrm{T}$ a insertar. En nues-

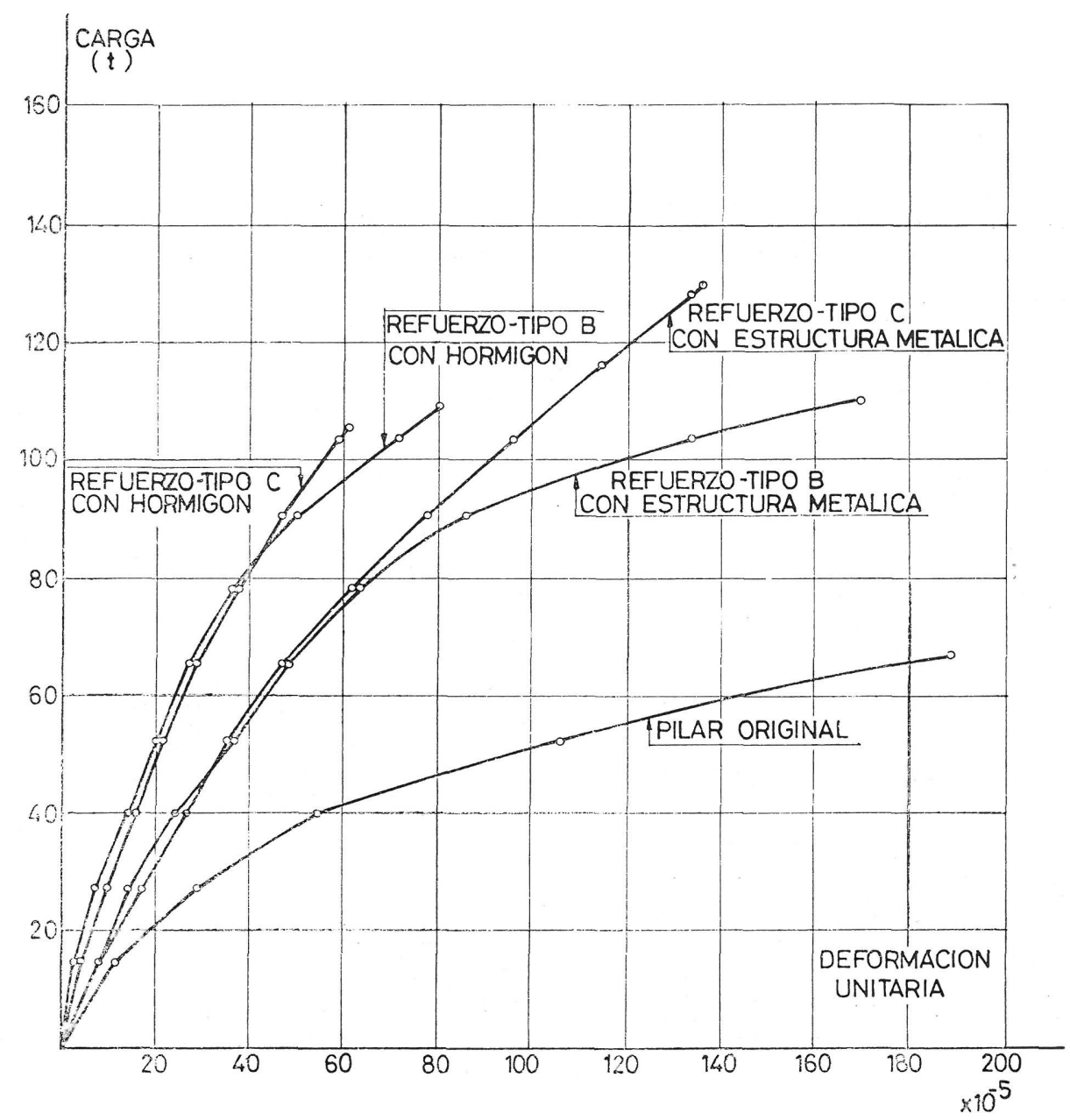


tro estudio, como ya se dijo, la cabeza de la doble T alcanza un tercio de la anchura del pilar $y$, merced a la mejora de resistencia por compresión localizada, transmite el $42 \%$ de la carga. El resto se transmite por el hormigón circundante sin alterar. El que la anchura de la doble $T$ sea una fracción de la del pilar representa que durante las obras de refuerzo no es necesario un gran apuntalamiento, ya que del orden de $2 / 3$ de la sección transversal del pilar quede intacta.

Las otras tres formas de refuerzo que hemos ensayado también podrán ser utilizadas, pero teniendo en cuenta su menor aficacia y la relación real entre sección de pilar y condiciones de aplastamiento y cortadura del forjado que se dispone.

En nuestro caso particular hemos dejado sin reforzar la zona que simula el forjado y pensamos que se nos ha producido la rotura en general por aplastamiento de dicha zona. Este efecto de aplastamiento se ha puesto de manifiesto en el caso de hormigón zunchado, en el que por es$\operatorname{tar} 1 \mathrm{~cm}$ separado el refuerzo del forjado no ha podido producirse fenómeno de cortadura y, sin embargo, las cargas de rotura son similares a las de los otros refuerzos.

En un caso real esta debilidad al aplastamiento hubiera sido menor debido a que la continuidad del forjado le produce una especie de zunchado, hecho que no ocurre en las piezas utilizadas en los ensayos.

De todas formas hay que pensar en que el aplastamiento y la cortadura de los forjados superior e inferior, con las adiciones de carga de pilar y de forjado que se dan en relación con el inferior, son precisamente las causantes de que no se alcance la resistencia intrínseca que aportan los refuerzos. Por ello estos problemas habrá que analizarlos con detalle garantizando la eficaz transmisión de cargas a través del refuerzo aislado o bien extendiendo el refuerzo, en caso de duda, a algún piso superior e inferior.

\section{BIBLIOGRAFIA}

1. SIDNEY, M. JOHNSON: "Deterioro, conservación y reparación de estructuras". Ed. Blume Labor, S. A., Madrid-Barcelona, 1973.

2. RAMIREZ, J. L., y BARCENA, J. M.: “Eficacia resistente de pilares de hormigón armado de baja calidad reforzados por dos procedimientos diferentes". INFORMES DE LA CONSTRUCCION, núm. 272, julio 1975. Colloquium RILEM, "Comportement en service des ouvrages en béton", Lieja, 4-6 junio 1975.

3. BERGHOLT, K., y HANSEN, T. C.: "Craking and repair of a reinforced concrete structure following reinforcement corrosion due to chloride contamination of aggregate". Colloquium RILEM, "Comportement en service des ouvrages en béton", Lieja, 4-6 junio 1975.

\section{résumé}

Comparaison de la résistance de quatre méthodes de renforcement des poteaux en béton armé

J. L. Ramírez Ortiz, Prof. Dr. ingénieur in dustriel

J. M. Bárcena Díaz, ingénieur industriel

J. M. Feijjóo Gil, ingénieur industriel

Ce travaíl, qui fait suite à celui paru dans le numéro 272 de cette revue (juillet 1975) est une étude du comportement résistan des poteaux en béton armé renforcés en étages isolés, par quatre autres procédés différents:

- Deux des méthodes de renforcement actuellement utilisées sont une amélioration de celles employées dans le travai anterieur. Elles consistent dans le fait de prolonger la section transversale avec du béton pénétrant dans des cavites pratiquées dans le béton primitif ou adosse de cornieres en acler aux aretes du poteau avec chapiteau de cornieres contre achésion du bóton ast de l'acier moyenaunt des résines.

- Les deux autres méthodes sont nouvelles dans notre expérimentation. Elles consis tent à créer une chemise extérieure de béton avec une armature ceinture et dans la solution métallique, à transpercer le poteau d'une poutre en double T qui transtère une partie de la double $T$ qui transfent à deux traverses plus re direcet, par conséquent, à rendre moins im portante la transmission d'effort par im chapiteau qui est toujours un peu souple.

\section{summary}

Stress comparison using four methods of reinforcement in reinforced concrete columns

J. M. Ramírez Ortiz, Prof. Dr. Industrial
Engineer

J. M. Bárcena Díaz, Industrial Engineer

J. M. Feijóo Gil, Industrial Engineer

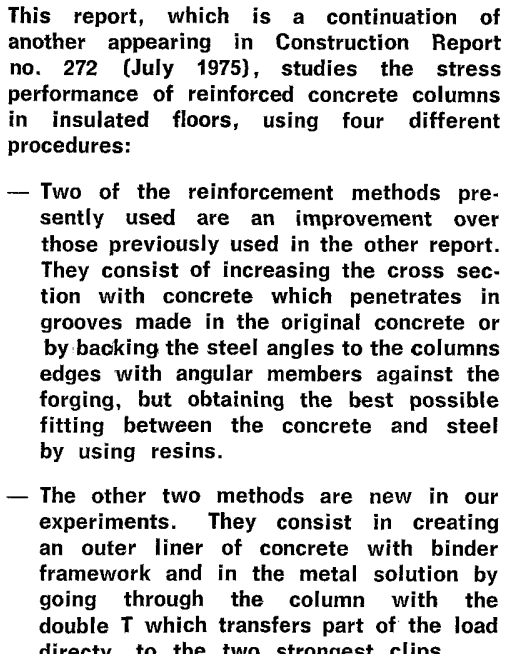

This report, which is a continuation of another appearing in Construction Report no. 272 (July 1975), studies the stress performance of reinforced concrete columns procedures:

Two of the reinforcement methods presently used are an improvement over the other report. They consist of increasing the cross sec. tion with concrete which penetrates in grooves made in the original concrete or by backing the steel angles to the columns edges with angular members against the fitting, but obtaining the best possible by using resins. outer liner of concrete with binder going through the column with the directy to the two strongest clips.

\section{zusammenfassung}

Vergleich von vier Methoden fuer Verstaerkung der Pfeiler aus armiertem Beton

J. L. Ramírez Ortiz, Prof. Dr. Maschineningenieur

J. M. Bárcena Díaz, Maschineningenieur

J. M. Feijóo Gil, Maschineningenieur

Diese Arbeit, die eine Forstsetzung der veröffentlichten in Information der Konstruktion Nr. 272 (Juli 1975) y studiert das Resistenzverua in vier anderen verschiedenen Verfahren.

in vier anderen verschiedenen Verfahren.

Zwei der Verstarkundt werden, sind eine Verbesserung der schon angewandten in der vorhergehenden Arbeit. Sie beruhen auf der Auftragung der transversalen Sektion mit Beton, der in die vorher eingearbeiteten Kasten im ursprunglichen Beton, eindringt oder auf dem Anbau von Winkeleisen an den Kanten de Pfeiler mit Säulen aufsatz mit Winkeleisen angebracht gegen die Stahlbetondecke. Dabei wird eine bessere Anpassung des Betons und des Stahis durch Harz erziehlt.

- Die anderen beiden Methoden sind neu in unserer Experimentation. Sie beruhen auf der Schaffung elnes ausseren Betonmantels mit einem Metalringgerüst und die Lösung wird aus Metal durchgeführt Die Trager werden mit einem doppe T-Stúck, das einen Teil der Belastung direkt auf zwei stäkere Stangen ubertragt, durchquert. Dadurch wird geringere ung des Säulenaufsatzes, das immer ung dexibler ist, zugemessen. 